\title{
TITLE:
}

\section{Electric resistance of magnetic domain wall in NiFe wires with CoSm pinning pads}

$\operatorname{AUTHOR}(S):$

Nagahama, T; Mibu, K; Shinjo, T

\section{CITATION:}

Nagahama, $T$...[et al]. Electric resistance of magnetic domain wall in NiFe wires with CoSm pinning pads. JOURNAL OF APPLIED PHYSICS 2000, 87(9): 5648-5650

\section{ISSUE DATE:}

2000-05-01

URL:

http://hdl.handle.net/2433/50359

\section{RIGHT:}

Copyright 2000 American Institute of Physics. This article may be downloaded for personal use only. Any other use requires prior permission of the author and the American Institute of Physics. 


\title{
Electric resistance of magnetic domain wall in NiFe wires with $\mathrm{CoSm}$ pinning pads
}

\author{
T. Nagahama, ${ }^{\text {a) }}$ K. Mibu, and T. Shinjo \\ Institute for Chemical Research, Kyoto University, Uji Kyoto-fu 611-0011, Japan
}

\begin{abstract}
The contribution of a magnetic domain wall to electric resistivity was measured using NiFe wires (width: $1 \mu \mathrm{m}$ ) partially covered with hard magnetic pads $(\mathrm{CoSm})$. When the wire is covered with $N$ pinning pads, $2 N$ domain walls can be produced in the wire by reversing the magnetization only at the uncovered parts. The resistance for the magnetically saturated state (no domain wall structure) and that for the magnetic structure with $2 \mathrm{~N}$ domain walls were compared at zero applied field. It was found that the resistance is smaller when magnetic domain walls exist, and that the domain wall resistance is almost temperature independent. (C) 2000 American Institute of Physics.
\end{abstract}

[S0021-8979(00)38608-X]

\section{INTRODUCTION}

Recently electric transport properties through magnetic domain walls have been attracting much attention. Gregg et al. ${ }^{1}$ studied the magnetoresistance of a Co thin film that has a stripe domain structure, and concluded that the resistance increases when domain walls exist due to an analogous effect with the giant magnetoresistance (GMR). Mibu et al. ${ }^{2}$ measured the magnetoresistance of a Bloch-wall-type magnetic structure using exchange-spring bilayers and observed a similar increase of resistance. Theoretically, Chang and Levy ${ }^{3}$ expanded the two current model used for understanding GMR to domain wall systems, and explained the increase of resistance by a magnetic domain wall. On the other hand, there are several reports that conclude that a domain wall causes a decrease in resistance. ${ }^{4-6}$ Hong et al. ${ }^{4}$ measured the resistance of a $\mathrm{Ni}$ nanowire and found that the resistance jumped down at the coercive field, where domain walls are nucleated. Tatara and Fukuyama ${ }^{7}$ proposed, from the weak localization regime, that decoherence of electrons due to a magnetic domain wall makes the resistivity smaller. Ruediger et $a l .{ }^{6}$ fabricated epitaxial $\mathrm{Fe}$ nanowires. The existence of a domain wall caused a decrease of resistance, and they attributed this decrease to suppression of surface scattering due to the change of Lorentz force direction in neighboring domains.

Here we report an experiment using NiFe wires partially pinned by hard magnetic CoSm pads. The wire is magnetically composed of two parts; one is free areas that have a small coercive field and the other is pinned areas that have a large coercive field. In a weak magnetic field, only the magnetization in the free parts changes the direction, and therefore $2 N$ magnetic domain walls are nucleated at the boundary between two different areas. (Here, $N$ is the number of pinning pads.) The resistance was compared for the no-wall state and $2 N$ wall state at zero external field. The difference of the resistance between the two states was also measured as a function of temperature.

a) Author to whom correspondence should be addressed; electronic mail: taro@jrcat.or.jp

\section{EXPERIMENT}

The sample was composed of a NiFe wire (thickness: $200 \AA$ A , width: $1 \mu \mathrm{m}$, length: $300 \mu \mathrm{m}$ ) and CoSm pinning pads. A schematic image of the sample is shown in Fig. 1(a). Every part, i.e., a NiFe wire, CoSm pads, and $\mathrm{Cu}$ electrodes, was prepared by a lift-off technique. The deposition and liftoff were repeated three times to prepare the sample. CoSm pads were made by alternate deposition of $\operatorname{Co}(5 \AA)$ and $\operatorname{Sm}(3$ $\AA$ ) with an external field of 600 Oe applied along the wire direction. This CoSm layer has an in-plane uniaxial anisotropy and a large coercive force. The resistance was measured by a conventional four-terminal method. The electrodes were

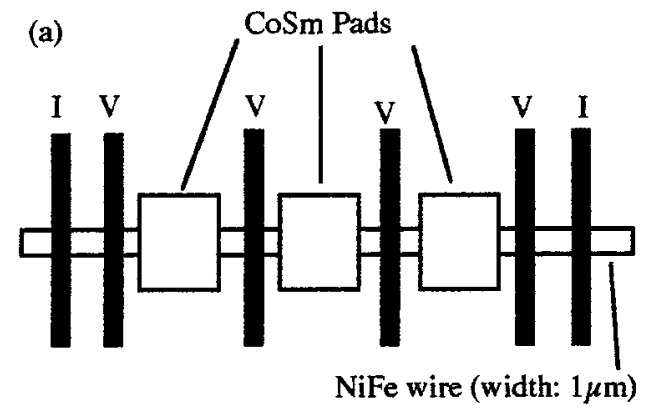

(b) No-Domain Wall

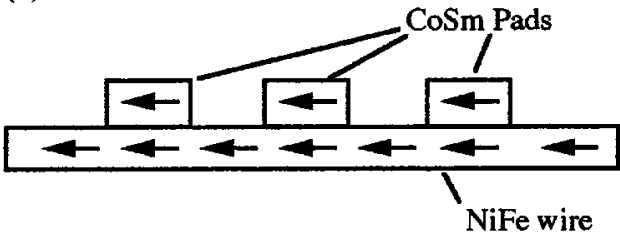

(c) $2 N$-Domain Wall

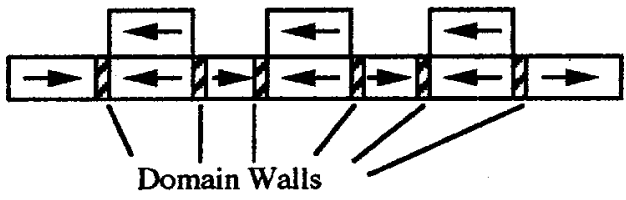

FIG. 1. The schematic image of: (a) a typical sample (top view), (b) no-wall state (magnetically saturated state)(side view), and (c) $2 N$ wall state (side view). 


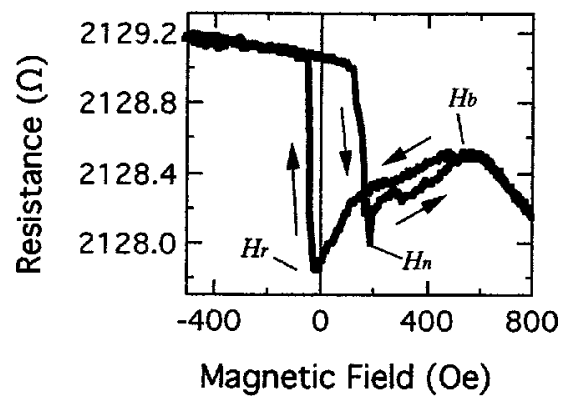

FIG. 2. The minor loop of the magnetoresistance of a NiFe (200 ̊) wire with three CoSm $(400 \AA)$ pads at $5 \mathrm{~K}$.

deposited at several points across the NiFe wire, so that we can choose the number of $\mathrm{CoSm}$ pads between the voltage terminals.

\section{RESULTS AND DISCUSSION}

Figure 2 shows a minor hysteresis loop of the magnetoresistance (MR) curve for a NiFe wire with three $\mathrm{CoSm}$ pinning pads. The starting point was the magnetic saturated state in a negative applied field [Fig. 1(b)]. When a magnetic field was applied to the opposite direction, the resistance decreased abruptly at a certain magnetic field $H_{n}$. Then, the resistance slightly increased, and started to decrease again at $H_{b}$. When the magnetic field was decreased from $800 \mathrm{Oe}$, the resistance first changed almost reversibly down to $H_{n}$. The resistance decreased slightly until the magnetic field went across zero, and returned to the saturated value abruptly at $H_{r}$. This change in MR curve is understood as follows: At $H_{n}$, antiparallel magnetic domains were nucleated in the $\mathrm{NiFe}$ wire, with the magnetic domain walls situated at the boundary between the pinned area and the free area [Fig. 1 (c)]. When the magnetic field exceeded $H_{b}$, the magnetic moments of the NiFe layer in the pinned area started to rotate gradually like soft magnetic layers in exchange-spring bilayers. ${ }^{8}$ When the magnetic field was reversed, the magnetization returned reversibly down to $H_{b}$. This spring magnetization process (i.e., reversible magnetization process) is evidence that the $\mathrm{NiFe}$ wire was magnetically pinned by the $\mathrm{CoSm}$ pads at the interface between $\mathrm{NiFe}$ and $\mathrm{CoSm}$. The magnetic domain walls remained down to $H_{r}$, and at $H_{r}$ they were annihilated. The reason why the resistance decrease from $H_{b}$ to $H_{r}$ is not clear, but that can be concerned with the magnetic structure of domain walls that changes slightly in this field range. If the magnetic structure does not change, the resistance should be kept constant.

It should be noticed that both no-wall state [Fig. 1(b)] and $2 N$ wall state [Fig. 1(c)] are realized at zero external field. Obviously there is a difference of resistance $\Delta R$ between the $2 N$ wall state and the no-wall state; the $2 N$ wall state has a smaller resistance than the no-wall state. This result shows that the domain wall contributes to negative MR. Figure 3 shows the dependence of $\Delta R$ on the wall number at $5 \mathrm{~K}$. A linear relation was obtained in the $\Delta R$ vs $N$ plot, and $\Delta R$ per wall was estimated to be about $0.17 \Omega$. We calculated the length of the domain wall under the assumption that $\Delta R$ is totally due to anisotropic magnetoresistance

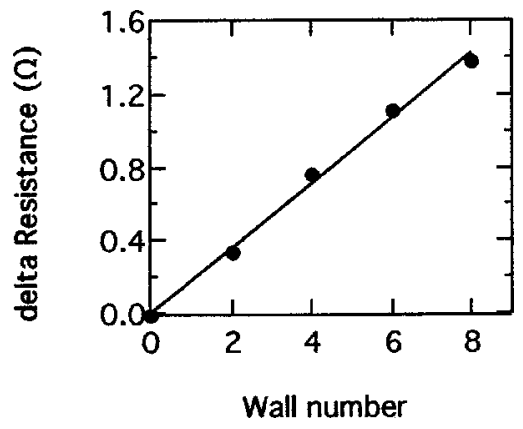

FIG. 3. $\Delta R$ vs number of domain walls.

(AMR) originating from the magnetic moments that make certain angles with the electric current direction. For simplicity, the angle of the magnetic moment in the wall was assumed to change linearly. Then the length of the domain wall was estimated to be about $1 \mu \mathrm{m}$. This value seems too long because usually the width of a domain wall is several hundreds $\AA$. However, in a head-on-head wall in a nanowire, a longer and complex domain wall structure was observed by magnetic force microscope (MFM) measurement. ${ }^{9}$ In this way, the observed decrease in magnetoresistance can be explained by the AMR effect in the domain walls.

Figure 4 shows the temperature dependence of $\Delta R$ for $\mathrm{NiFe}$ wires with 1-4 pinning pads. Here, the ordinary magnetoresistance observed for epitaxial $\mathrm{Fe}$ wires ${ }^{6}$ is negligible, because the mean free path is not so long in these samples. The values of $\Delta R$ did not depend on temperature and this tendency is similar to that of AMR. This is more evidence that the difference of the resistance at zero field is mainly due to the AMR effect in the magnetic domain walls.

\section{SUMMARY}

We fabricated NiFe wires partially covered by CoSm pinning pads, and compared the resistance between the nowall state and the wall-existing state at zero field. The magnetic domain wall decreased the resistance by $0.17 \Omega$ per wall and the effect did not depend on temperature. The decrease can be attributed to the AMR effect with the assumption that the length of the wall is about $1 \mu \mathrm{m}$.

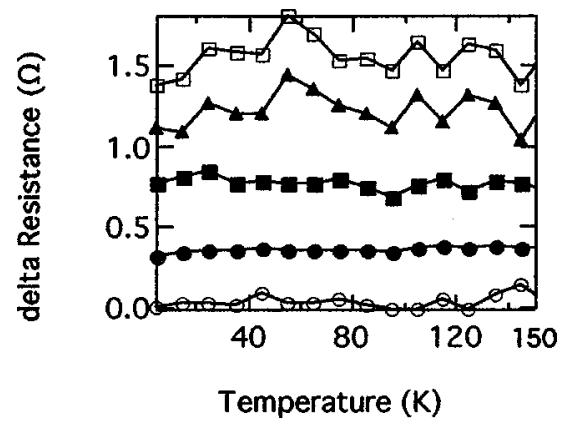

FIG. 4. The plot of temperature dependence of $\Delta R$ for the NiFe wire with: $(\bigcirc)$ : zero, $(\mathbf{O})$ : one, $(\boldsymbol{\square})$ : two, $(\boldsymbol{\Delta})$ : three, and $(\square)$ : four pinning pads. 


\section{ACKNOWLEDGMENTS}

This work was partially supported by a Grant-in-Aid for Scientific Research on Priority Area from Monbusho, and JSPS Research Fellowships for Young Scientists.

${ }^{1}$ J. F. Gregg, W. Allen, K. Ounadjela, M. Viret, M. Hehn, S. M. Thompson, and J. M. D. Coey, Phys. Rev. Lett. 77, 1580 (1996).

${ }^{2}$ K. Mibu, T. Nagahama, and T. Shinjo, Phys. Rev. B 58, 6442 (1998).

${ }^{3}$ P. M. Levy and S. Zhang, Phys. Rev. Lett. 79, 5110 (1997).
${ }^{4}$ K. Hong and N. Giordano, J. Phys.: Condens. Matter 10, L401 (1998).

${ }^{5}$ Y. Otani, S. G. Kim, K. Fukamichi, O. Kitakami, and Y. Shimada, IEEE Trans. Magn. 34, 1096 (1998).

${ }^{6}$ U. Ruediger, J. Yu, S. Zhang, A. D. Kent, and S. S. P. Parkin, Phys. Rev. Lett. 80, 5639 (1998).

${ }^{7}$ G. Tatara and H. Fukuyama, Phys. Rev. Lett. 78, 3773 (1998).

${ }^{8}$ T. Nagahama, K. Mibu, and T. Shinjo, J. Phys. D: Appl. Phys. 31, 43 (1998)

${ }^{9}$ Y. Nozaki, K. Matsuyama, T. Ono, and H. Miyajima, Jpn. J. Appl. Phys., Part 1 38, 6282 (1999). 